\title{
Association of cesarean section and allergic outcomes among infants at 1 year of age: Logistics regression analysis using data of 104,065 fetal and children's records from the Japan Environment and Children's Study
}

\author{
Hajime Maeda $^{1}$, Koichi Hashimoto ${ }^{1}$, Hajime Iwasa ${ }^{1}$, Hyo Kyozuka ${ }^{1}$, Hayato Go ${ }^{1}$, Akiko \\ Sato $^{2}$, Yuka Ogata ${ }^{3}$, Tsuyoshi Murata ${ }^{1}$, Keiya Fujimori ${ }^{1}$, Kosei Shinoki ${ }^{3}$, Hidekazu \\ Nishigori $^{1}$, Seiji Yasumura ${ }^{1}$, and Mitsuaki Hosoya ${ }^{1}$ \\ ${ }^{1}$ Fukushima Medical University School of Medicine \\ ${ }^{2}$ Fukushima Regional Center for the Japan Environmental and Children's Study \\ ${ }^{3}$ Fukushima Regional Center for the Japan Environmental and Children's Study
}

March 9, 2021

\begin{abstract}
Background: Globally, the incidence of allergic diseases is increasing along with cesarean delivery rates. Data regarding the association between cesarean delivery and allergic diseases are inconsistent. Here, we aimed to clarify the association between cesarean delivery and the development of asthma, atopic dermatitis, wheezing, and eczema. Methods: We used data from the Japan Environment and Children's Study. The data included were those of 104,065 fetal records and their children. Information about the mode of delivery, asthma, atopic dermatitis, wheezing, and eczema was obtained from questionnaires and medical record transcripts. Multiple logistic regression analysis was used to assess the association between cesarean delivery and asthma, atopic dermatitis, wheezing, and eczema risk among infants at 1 year of age. Results: We included 74,639 subjects in this study, wherein $18.4 \%$ underwent cesarean deliveries. After adjusting for the perinatal, socioeconomic, and postnatal confounding factors, children born by cesarean delivery had no increased risk of developing asthma [95\% confidence interval (CI) 0.84-1.08], atopic dermatitis (95\% CI, 0.92-1.13), wheezing (95\% CI, 0.94-1.04), or eczema (95\% CI, 0.94-1.05). Conclusions: This nationwide cohort study found no association between cesarean delivery and asthma, atopic dermatitis, wheezing, and eczema among infants at 1 year of age. However, further studies are needed to evaluate whether cesarean delivery plays a role in the development of allergic diseases.
\end{abstract}

Association of cesarean section and allergic outcomes among infants at 1 year of age: Logistics regression analysis using data of 104,065 fetal and children's records from the Japan Environment and Children's Study

Hajime Maeda ${ }^{1}$, Koichi Hashimoto ${ }^{1,2}$, Hajime Iwasa ${ }^{3}$, Hyo Kyozuka ${ }^{2,4}$, Hayato Go ${ }^{1}$, Akiko Sato ${ }^{2}$, Yuka Ogata $^{2}$, Tsuyoshi Murata ${ }^{2,4}$, Keiya Fujimori ${ }^{2,4}$, Kosei Shinoki ${ }^{2}$, Hidekazu Nishigori ${ }^{2,5}$, Seiji Yasumura ${ }^{2,3}$, Mitsuaki Hosoya ${ }^{1,2}$, and the Japan Environment and Children's Study (JECS) Group

${ }^{1}$ Department of Pediatrics, School of Medicine, Fukushima Medical University, Fukushima, Japan ${ }^{2}$ Fukushima Regional Center for the Japan Environmental and Children's Study, Fukushima, Japan; ${ }^{3}$ Department of Public Health, School of Medicine, Fukushima Medical University, Fukushima, Japan; ${ }^{4}$ Department of Obstetrics and Gynecology, School of Medicine, Fukushima Medical University School, Fukushima, Japan; and ${ }^{5}$ Fukushima Medical Center for Children and Women, Fukushima Medical University, Fukushima, Japan 
Running title: Cesarean delivery and allergic diseases

\section{Corresponding author:}

Hajime Maeda

Department of Pediatrics, School of Medicine, Fukushima Medical University

1 Hikarigaoka, Fukushima 960-1295, Japan

Telephone number: 81-245-47-1111

Fax number: 81-245-47-1998

Email address: hmaeda@fmu.ac.jp

Word count: 2479, Tables: 3, Figure: 1

i. Statement of potential conflicts of interests:

The authors have no conflicts of interest to declare.

\section{ii. Financial support:}

This study was funded by the Ministry of the Environment, Japan. The findings and conclusions of this article are solely the responsibility of the authors and do not represent the official views of the above government.

\section{Key message}

In a large nationwide cohort study, we did not find an association between cesarean delivery and asthma, atopic dermatitis, wheezing, and eczema among infants at 1 year of age.

\section{iii. Abstract:}

Background: Globally, the incidence of allergic diseases is increasing along with cesarean delivery rates. Data regarding the association between cesarean delivery and allergic diseases are inconsistent. Here, we aimed to clarify the association between cesarean delivery and the development of asthma, atopic dermatitis, wheezing, and eczema.

Methods: We used data from the Japan Environment and Children's Study. The data included were those of 104,065 fetal records and their children. Information about the mode of delivery, asthma, atopic dermatitis, wheezing, and eczema was obtained from questionnaires and medical record transcripts. Multiple logistic regression analysis was used to assess the association between cesarean delivery and asthma, atopic dermatitis, wheezing, and eczema risk among infants at 1 year of age.

Results: We included 74,639 subjects in this study, wherein $18.4 \%$ underwent cesarean deliveries. After adjusting for the perinatal, socioeconomic, and postnatal confounding factors, children born by cesarean delivery had no increased risk of developing asthma [95\% confidence interval (CI) 0.84-1.08], atopic dermatitis (95\% CI, 0.92-1.13), wheezing (95\% CI, 0.94-1.04), or eczema (95\% CI, 0.94-1.05).

Conclusions: This nationwide cohort study found no association between cesarean delivery and asthma, atopic dermatitis, wheezing, and eczema among infants at 1 year of age. However, further studies are needed to evaluate whether cesarean delivery plays a role in the development of allergic diseases.

\section{Keywords :}

cesarean delivery, allergic disease, atopic dermatitis, asthma, wheezing, eczema

iv. Main text:

Introduction 
The incidence of allergic diseases has recently increased in different regions around the world. ${ }^{1}$ In Japan, allergic diseases are a serious health concern, with high rates of wheezing (16.4\%), asthma (10.5\%), and atopic dermatitis $(21.5 \%)$ observed in children at 5 years of age. ${ }^{2}$ Concurrently, cesarean delivery rates have been increasing worldwide, ${ }^{3-5}$ and have approximately doubled in Japan since 1990, reaching $18.6 \%$ in $2014 .{ }^{6}$ The increased rate is mainly due to a rise in elective cesarean delivery. Some recent reports describe associations between cesarean delivery and allergic diseases. ${ }^{7-9}$ Given that the increases in allergic disease rates have occurred over a short period of time, they may be associated with environmental factors rather than genetic factors. Compared with children born by vaginal delivery, those born by cesarean delivery have different gut flora and cytokine profiles, ${ }^{10-12}$ which are thought to be related to allergic diseases. In contrast, some reports showed that delivery by cesarean section was not associated with the development of allergic diseases. ${ }^{13-15}$ Therefore, the association between cesarean delivery and allergic diseases is controversial. In this study, we investigated the association between infants born by cesarean delivery and the development of asthma, atopic dermatitis, wheezing, and eczema using data from a large sample size cohort study of the Japan Environment and Children's Study (JECS).

\section{Methods}

\section{Study design}

In the present study, we used data from the JECS, which is a nationwide, government-funded birth cohort study. ${ }^{16}$ The JECS investigates the effects of environmental factors on children's health by tracking mothers and their children until the children reach 13 years of age. From January 2011 to March 2014, 103,062 pregnancies were included in the JECS at 15 Regional Centres in Japan (Hokkaido, Miyagi, Fukushima, Chiba, Kanagawa, Koshin, Toyama, Aichi, Kyoto, Osaka, Hyogo, Tottori, Kochi, Fukuoka, and South Kyushu/Okinawa). Caregivers completed questionnaires regarding the mothers and their children during pregnancy and when the children were 6 and 12 months of age. The JECS protocol was reviewed and approved by the Ministry of the Environment's Institutional Review Board on Epidemiological Studies and Ethics Committees of all participating institutions. The JECS was conducted in accordance with the Declaration of Helsinki and other nationally valid regulations and guidelines. Written informed consent was obtained from all participants.

\section{Data collection}

We used seven types of data from the dataset released in March 2018 (jecs-an-20180131) that included information from questionnaires and medical record transcripts. MT1 provided data from self-reported questionnaires collected during the first trimester that included information about maternal medical backgrounds. DrT1 provided medical information collected from the medical record transcripts during the first trimester provided by each co-operating health care provider. MT2 provided data from self-reported questionnaires collected during the second/third trimester that included information on lifestyle and socioeconomic status. Dr0m provided perinatal information collected from the medical record transcripts provided by each co-operating health care provider. M1m provided data from self-reported questionnaires collected at one month after birth. C6m provided data from self-reported questionnaires collected at six months after birth. C1Y provided data from self-reported questionnaires collected at 12 months after birth. We excluded women who miscarried, had multiple births, whose babies were stillborn, or whose data regarding confounders were missing (Fig. 1).

\section{Outcomes, exposures, and covariates}

Asthma, atopic dermatitis, wheezing, and eczema were assessed based on the doctors' self-reported diagnoses and the information given in the participants' questionnaires when the children were 1 year old (C1Y data). The questionnaire included whether wheezing and eczema occurred in the first year of life. This was a validated, modified International Study of Asthma and Allergies in Childhood (ISAAC) questionnaire for children 6-7 years old, translated in Japanese. ${ }^{17-19}$ The subjects were assigned to a cesarean or vaginal delivery group, based on the modes of delivery recorded in the Dr0m data. Moreover, participants in the cesarean delivery group were assigned to an elective or emergency group based on the reason for cesarean 
delivery recorded in the Dr0m data: elective cesarean delivery included repeat cesarean delivery, history of uterine surgery, placenta previa, and fetal malpresentation and emergency cesarean delivery included gestational hypertension syndrome, nonreassuring fetal status, delayed/obstructed labor, early rupture of membrane, intrauterine infection, cephalopelvic disproportion, and complication/other reason. We considered the following factors as possible confounders in the regression analyses: maternal age at pregnancy, parity, sex, gestational age (GA), small for gestational age (SGA), maternal allergy history, maternal active and passive smoking during pregnancy, maternal educational levels, annual family income, marital status, breastfeeding at six months, pet ownership, and passive smoking exposure of infants after birth. The maternal age at pregnancy (DrT1 data) was categorized as follows: < 20, 20-29, 30-39, and [?] 40 years. Parity (DrT1 data) was categorized as 0,1 , or [?] 2. The GA was categorized as follows: $<37,37,38$, and 39-41 weeks. Standard deviation (SD) was calculated based on Japanese neonatal anthropometric charts, ${ }^{20}$ which accounted for GA, sex, and parity. Babies with values $<-1.5$ SDs were defined as SGA. ${ }^{21}$ Maternal allergy history (MT1 data) was considered positive if a parent reported a history of asthma, allergic rhinitis, atopic dermatitis, allergic conjunctivitis, food allergies, drug allergies, contact dermatitis, or sick building syndrome. Female participants were asked to provide information about their smoking status (MT2 data), as follows: "Never", "Previously did, but quit before recognizing current pregnancy", "Previously did, but quit after finding out current pregnancy", and "Yes, I still smoke". The female participants whose responses were "Yes, I still smoke" were assigned to the smoking category, and the remaining participants were assigned to the non-smoking category.

Maternal educational periods (MT2 data) were grouped as follows: junior high school, $<10$ years; high school, 10-12 years; technical/vocational college or university, 13-16 years; and graduate school, [?] 17 years. The participants' annual household incomes (MT2 data) were categorized as follows: < 2,000,000 Japanese yen (JPY); 2,000,000-5,999,999 JPY; 6,000,000-9,999,999 JPY; and [?] 10,000,000 JPY. Data on marital status were obtained from MT1. Data on passive smoking exposure of infants were obtained from M1m. Data on breastfeeding and pet ownership were obtained from C6m.

\section{Statistical analyses}

The characteristics of the mothers and their children were summarized according to the delivery mode. A chi-squared test was used to compare categorical variables. Multiple logistic regression was performed to determine the risks of asthma, atopic dermatitis, wheezing, and eczema associated with cesarean delivery by calculating the odds ratios (ORs), which were adjusted for the aforementioned confounders, and $95 \%$ confidence intervals (CIs). We initially adjusted for perinatal and socioeconomic factors. We then adjusted for perinatal, socioeconomic, and postnatal factors. Finally, we estimated the association between elective and emergency cesarean delivery (vs. vaginal delivery) and the risks of asthma, atopic dermatitis, wheezing, and eczema. Statistical analyses were performed using Stata, version 15.0 (Stata Corporation LLC, College Station, TX, USA). $P$-values $<0.05$ were considered statistically significant.

\section{Results}

The jecs-an-20180131 dataset contained information about 104,065 fetuses and 98,255 live singleton births. After applying the study's inclusion criteria, 74,639 subjects were eligible to participate in this study (Fig. 1). Of the 74,639 mothers, $13,756(18.4 \%)$ and $60,883(81.6 \%)$ underwent cesarean and vaginal delivery, respectively. Of the 13,756 cesarean deliveries, $8,057(10.8 \%)$ and 5,699 (7.6\%) were elective and emergency cesarean deliveries, respectively. Table 1 presents the participants' characteristics. A higher incidence of cesarean delivery was associated with a maternal age at pregnancy [?] 30 years, parity [?] 2, GA < 39 weeks, SGA, maternal smoking during pregnancy, higher maternal education, higher annual family income, and pet ownership. A lower incidence of cesarean delivery was associated with primipara and breastfeeding at six months. The rates of asthma, atopic dermatitis, wheezing, and eczema in those born via cesarean delivery were $2.7 \%, 4.2 \%, 20.2 \%$, and $18.1 \%$, respectively; while the rates for those born via vaginal delivery were $2.5 \%, 4.4 \%, 19.5 \%$, and $19.0 \%$, respectively. There were differences noted in the rate of eczema between the cesarean and vaginal delivery groups, while there were no differences in the rates of asthma, atopic dermatitis, and wheezing. 
Multiple logistic regression was performed to assess the risks of asthma, atopic dermatitis, wheezing, and eczema associated with cesarean delivery (Table 2). After adjusting for perinatal and socioeconomic confounding factors, cesarean delivery was not associated with an increased risk of asthma [adjusted ORs (aOR) $=0.97 ; 95 \% \mathrm{CI}, 0.85-1.10]$, atopic dermatitis $(\mathrm{aOR}=1.02 ; 95 \% \mathrm{CI}, 0.92-1.13)$, wheezing $(\mathrm{aOR}=0.99 ; 95 \%$ $\mathrm{CI}, 0.94-1.04$ ), or eczema (aOR $=0.99 ; 95 \% \mathrm{CI}, 0.94-1.04)$. After adjusting for perinatal, socioeconomic, and postnatal confounding factors, cesarean delivery was not associated with an increased risk of asthma $(\mathrm{aOR}=0.95 ; 95 \% \mathrm{CI}, 0.84-1.08)$, atopic dermatitis $(\mathrm{aOR}=1.02 ; 95 \% \mathrm{CI}, 0.92-1.13)$, wheezing $(\mathrm{aOR}=$ 0.98; 95\% CI, 0.94-1.04), or eczema (aOR $=0.99 ; 95 \%$ CI, 0.94-1.05). Overall crude ORs and aORs for the association between elective and emergency cesarean delivery (vs. vaginal delivery) and the risks of asthma, atopic dermatitis, wheezing, and eczema are shown in Table 3. After adjusting for perinatal and socioeconomic confounding factors, elective cesarean delivery was associated with an increased risk of wheezing (aOR $=1.07 ; 95 \% \mathrm{CI}, 1.01-1.13)$ and emergency cesarean delivery was associated with a decreased risk of eczema $(\mathrm{aOR}=0.90 ; 95 \% \mathrm{CI}, 0.84-0.97)$. After adjusting for perinatal, socioeconomic, and postnatal confounding factors, emergency cesarean delivery was associated with a decreased risk of eczema (aOR $=0.91 ; 95 \% \mathrm{CI}$, $0.84-0.97)$.

\section{Discussion}

In this study, no associations were found between cesarean delivery and the development of asthma, atopic dermatitis, wheezing, and eczema among infants at 1 year of age. Moreover, patients delivered through elective and emergency cesarean deliveries had no increased risk of asthma, atopic dermatitis, wheezing, and eczema after adjusting for perinatal, socioeconomic, and postnatal confounding factors. Numerous studies have investigated the associations between cesarean delivery and allergic diseases, but the results have been inconsistent. The findings from a previous meta-analysis that included 15 cohort studies, four case-control studies, and one cross-sectional study, showed an association between cesarean delivery and asthma, and an increased risk of asthma among children born by cesarean delivery $(\mathrm{OR}=1.20 ; 95 \% \mathrm{CI}$, 1.14-1.26). ${ }^{7}$ Moreover, the authors of a meta-analysis that included 23 cohort studies and three case-control studies concluded that allergic outcomes were attributable to cesarean delivery in only $1-4 \%$ of subjects. ${ }^{8}$ In contrast, a population-based cohort study in the UK in 2004 showed that cesarean delivery was not associated with the subsequent development of physician-diagnosed asthma, wheezing, or atopy in later childhood $(\mathrm{aOR}=1.14 ; 95 \% \mathrm{CI}, 0.9-1.4 ; \mathrm{aOR}=0.95 ; 95 \% \mathrm{CI}, 0.7-1.3]$; and $\mathrm{aOR}=1.04 ; 95 \% \mathrm{CI}, 0.8-1.3$, respectively). ${ }^{13}$ A population-based cohort study in the USA in 2005 showed that the mode of delivery was not associated with subsequent risk of developing childhood asthma (adjusted hazard ratio: 0.93; $95 \%$ CI, 0.6-1.4) or wheezing episodes (adjusted hazard ratio: 0.93; 95\% CI, 0.7-1.3). ${ }^{14}$ A recent cohort study in Taiwan in 2017 showed that asthma was not associated with cesarean delivery after controlling for GA and parental history of asthma $(\mathrm{aOR}=1.11 ; 95 \% \mathrm{CI}, 0.98-1.25) .{ }^{15}$ Previous studies examining delivery by cesarean section and the development of infectious diseases had variable sample sizes, age groups, follow-up times, case definitions, and adjustments for confounding factors. However, this study is the largest pregnancy cohort study in which the association between cesarean delivery and asthma, atopic dermatitis, wheezing, and eczema among infants at 1 year of age was assessed while controlling for a wide range of influencing factors.

Children born by cesarean delivery show delays and differences in relation to the establishment of their gut flora, as well as altered cytokine profiles. ${ }^{10-12}$ Infants born by cesarean delivery acquire a microbiota that differs from that in infants delivered vaginally. While infants delivered vaginally harbor bacterial communities that resemble those of their mothers' vaginas, infants born by cesarean delivery are enriched with skin microbiota. ${ }^{10,11}$ Hence, the delivery mode may be a crucial factor that influences the development of an infant's immune system and subsequent incidence of disease. Altered perinatal toll-like receptor responses and aberrant changes in cord blood cytokine responses, including those associated with interleukin (IL)-6, IL8 , IL-10, IL-13, tumor necrosis factor- $\alpha$, and interferon- $\gamma$, are related to asthma, and neonates with bacterial colonization of the airways are at an increased risk of developing recurrent wheezing and asthma in early life. ${ }^{12}$ Another mechanism may be changes in the stress hormone levels at birth between cesarean and vaginal deliveries because infants delivered by cesarean section before the onset of labor lack the normal surge of 
stress hormones. ${ }^{22}$ These mechanisms may affect elective cesarean deliveries more than emergency cesarean deliveries because emergency cesarean deliveries often occur after the onset of labor, potentially resulting in exposure to the vaginal microflora and both maternal and fetal stress. ${ }^{22}$ Children born by cesarean delivery, especially elective cesarean delivery, may have an increased risk of developing allergic diseases. However, the results from our study do not support this hypothesis. Based on recent evidence of the presence of bacteria in the placenta, amniotic fluid, and meconium, some investigators posit that the microbiome may be seeded before birth. ${ }^{23}$ These findings support the results from our study that showed there were no associations between cesarean delivery and the development of allergic diseases. Interestingly, in this study, emergency cesarean delivery reduced the development of eczema. However, this mechanism is unclear.

The strengths of our study include the prospective and nationwide design; the comparison of maternal questionnaires and medical records with birth certificates to verify the main exposure variable and other covariates; and use of prospectively collected data on asthma, atopic dermatitis, wheezing, and eczema. Multiple regression analysis was performed to correct for perinatal, socioeconomic, and postnatal factors.

However, this study has several limitations. First, we evaluated allergic diseases at 1 year of age using participants' self-reported questionnaires, which may have led to the under-reporting of allergic diseases. Second, many infants may not be diagnosed with allergic diseases at 1 year of age because the diagnostic criteria for allergic diseases at this age are not clear. Finally, cesarean delivery may not have been strictly assigned to the elective or emergency cesarean delivery group in the JECS data; however, emergency cesarean delivery may have also included elective cesarean delivery. Most elective cesarean deliveries assigned in this study occurred before the onset of labor and exposure to vaginal flora. Despite these limitations, our study evaluated data from a large, nationwide, prospective birth cohort study, and therefore, provides strong evidence against an association between cesarean delivery and allergic diseases. This may have important clinical and public health implications. If cesarean delivery has clinical benefits, it should not be avoided because of the risk of allergic diseases in infants.

\section{Conclusions}

Our findings, which were based on a large nationwide cohort, revealed no association between cesarean delivery and the development of allergic disease. Further studies are needed to evaluate whether cesarean delivery plays a role in the development of allergic diseases.

\section{v. Acknowledgements:}

The findings and conclusions of this article are solely the responsibility of the authors and do not represent the official views of the Ministry of the Environment, Japan. We thank all the participants and staff involved in the JECS. Members of the JECS Group as of 2020: Michihiro Kamijima (principal investigator, Nagoya City University, Nagoya, Japan), Shin Yamazaki (National Institute for Environmental Studies, Tsukuba, Japan), Yukihiro Ohya (National Center for Child Health and Development, Tokyo, Japan), Reiko Kishi (Hokkaido University, Sapporo, Japan), Nobuo Yaegashi (Tohoku University, Sendai, Japan), Koichi Hashimoto (Fukushima Medical University, Fukushima, Japan), Chisato Mori (Chiba University, Chiba, Japan), Shuichi Ito (Yokohama City University, Yokohama, Japan), Zentaro Yamagata (University of Yamanashi, Chuo, Japan), Hidekuni Inadera (University of Toyama, Toyama, Japan), Takeo Nakayama (Kyoto University, Kyoto, Japan), Hiroyasu Iso (Osaka University, Suita, Japan), Masayuki Shima (Hyogo College of Medicine, Nishinomiya, Japan), Youichi Kurozawa (Tottori University, Yonago, Japan), Narufumi Suganuma (Kochi University, Nankoku, Japan), Koichi Kusuhara (University of Occupational and Environmental Health, Kitakyushu, Japan), and Takahiko Katoh (Kumamoto University, Kumamoto, Japan).

\section{Authors' contributions}

H.M. conceptualized and designed the study, analyzed the data, and drafted the initial manuscript. K.H., H.I., and H.K. contributed to the data analysis and interpretation and assisted in the preparation of the manuscript, giving critical advice. H.G. and T.M. gave advice on the data analysis. A.S. and Y.O. collected the data. K.F., H.N., S.Y., M.H., and the JECS group reviewed the manuscript and gave critical advice. 
All of the authors discussed the results and contributed to the final manuscript. All of the authors read and approved the final manuscript.

\section{vi. Impact statement:}

Our study evaluated data from a large, nationwide, prospective birth cohort study and revealed no association between cesarean delivery and the development of allergic disease among infants at 1 year of age.

\section{vii. References}

1. Wong GW, Leung TF, Ko FW. Changing prevalence of allergic diseases in the Asia-pacific region. Allergy Asthma Immunol Res 2013;5:251-257.

2. Yamamoto-Hanada K, Yang L, Narita M, Saito H, Ohya Y. Influence of antibiotic use in early childhood on asthma and allergic diseases at age 5. Ann Allergy Asthma Immunol 2017;119:54-58.

3. Menacker F, Hamilton BE. Recent trends in cesarean delivery in the United States. NCHS Data Brief 2010;35:1-8.

4. Finger C. Caesarean section rates skyrocket in Brazil. Many women are opting for caesareans in the belief that it is a practical solution. Lancet 2003;362:628.

5. Feng XL, Xu L, Guo Y, Ronsmans C. Factors influencing rising caesarean section rates in China between 1988 and 2008. Bull World Health Organ 2012;90:30-39.

6. Maeda E, Ishihara O, Tomio J, et al. Cesarean section rates and local resources for perinatal care in Japan: a nationwide ecological study using the national database of health insurance claims. J Obstet Gynaecol Res 2018;44:208-216.

7. Thavagnanam S, Fleming J, Bromley A, Shields MD, Cardwell CR. A meta-analysis of the association between Caesarean section and childhood asthma. Clin Exp Allergy 2008;38:629-633.

8. Bager P, Wohlfahrt J, Westergaard T. Caesarean delivery and risk of atopy and allergic disease: metaanalyses. Clin Exp Allergy 2008;38:634-642.

9. Chu S, Chen Q, Chen Y, Bao Y, Wu M, Zhang J. Cesarean section without medical indication and risk of childhood asthma, and attenuation by breastfeeding. PLoS One 2017;12:e0184920.

10. Dominguez-Bello MG, Costello EK, Contreras M, et al. Delivery mode shapes the acquisition and structure of the initial microbiota across multiple body habitats in newborns. Proc Natl Acad Sci U S A 2010;107:11971-11975.

11. Dominguez-Bello MG, De Jesus-Laboy KM, Shen N, et al. Partial restoration of the microbiota of cesarean-born infants via vaginal microbial transfer. Nat Med 2016;22:250-253.

12. Liao SL, Tsai MH, Yao TC, et al. Caesarean section is associated with reduced perinatal cytokine response, increased risk of bacterial colonization in the airway, and infantile wheezing. Sci Rep 2017;7:9053.

13. Maitra A, Sherriff A, Strachan D, Henderson J, ALSPAC Study Team. Mode of delivery is not associated with asthma or atopy in childhood. Clin Exp Allergy 2004;34:1349-1355.

14. Juhn YJ, Weaver A, Katusic S, Yunginger J. Mode of delivery at birth and development of asthma: a population-based cohort study. J Allergy Clin Immunol 2005;116:510-516.

15. Chen G, Chiang WL, Shu BC, Guo YL, Chiou ST, Chiang TL. Associations of caesarean delivery and the occurrence of neurodevelopmental disorders, asthma or obesity in childhood based on Taiwan birth cohort study. BMJ Open 2017;7:e017086.

16. Kawamoto T, Nitta H, Murata K, et al. Rationale and study design of the Japan environment and children's study (JECS). BMC Public Health 2014;14:25. 
17. Asher MI, Keil U, Anderson HR, et al. International Study of Asthma and Allergies in Childhood (ISAAC): Rationale and methods. Eur Respir J 1995;8;483-491

18. Weiland SK, Björkstén B, Brunekreef B, Cookson WO, von Mutius E, Strachan DP, and the ISAAC Phase Two Study Group. Phase II of the International Study of Asthma and Allergies in Childhood (ISAAC II): rationale and methods. Eur Respir J 2004; 24(3): 406-12.

19. Ellwood P, Asher MI, Beasley R, Clayton TO, Stewart AW, and the ISAAC Steering Committee. The International Study of Asthma and Allergies in Childhood (ISAAC): Phase Three rationale and methods. Int J Tuberc Lung Dis 2005; 9(1): 10-6.

20. Itabashi K, Miura F, Uehara R, Nakamura Y. New Japanese neonatal anthropometric charts for gestational age at birth. Pediatr Int 2014;56;702-708.

21. Kyozuka H, Fujimori K, Hosoya M, et al. The effect of maternal age at the first childbirth on gestational age and birth weight: The Japan Environment and Children's Study (JECS). J Epidemiol. 2019;29;187-191.

22. Rusconi F, Zugna D, Annesi-Maesano I, et al. Mode of Delivery and Asthma at School Age in 9 European Birth Cohorts. Am J Epidemiol. 2017;185;465-473.

23. Willyard C. Could baby's first bacteria take root before birth? Nature 2018;553:264-266.

\section{viii. Tables:}

Table 1. Characteristics of the study participants.

\begin{tabular}{lll}
\hline & Vaginal delivery $(\mathrm{n}=60,883)$ & $\%$ \\
\hline & $\mathrm{n}$ & \\
Maternal age at pregnancy (years) & & 0.7 \\
$<20$ & 405 & 40.1 \\
$20-29$ & 24,436 & 56.3 \\
$30-39$ & 34,254 & 2.9 \\
$>=40$ & 1,788 & 29.8 \\
Parity & & 34.7 \\
0 & 18,167 & 35.4 \\
1 & 21,140 & 51.1 \\
$>=2$ & 21,576 & 3.0 \\
Sex (male) & 31,124 & 6.1 \\
Gestational age (weeks) & & 18.1 \\
37 & 1,834 & 72.8 \\
38 & 3,738 & 3.1 \\
$39-41$ & 11,012 & 51.6 \\
SGA & 44,299 & 3.7 \\
Maternal allergies & 1,882 & 35.6 \\
passive smoking during pregnancy & 31,399 & 3.7 \\
$<10$ & 2,227 & 31.1 \\
$10-12$ & 21,644 & 63.6 \\
$13-16$ & & 1.6
\end{tabular}




\begin{tabular}{lll}
\hline & Vaginal delivery $(\mathrm{n}=60,883)$ & \\
\hline $6,000,000-9,999,999$ & 13,954 & 22.9 \\
$>=10,000,000$ & 2,593 & 4.3 \\
Marriage & 58,786 & 96.6 \\
Breastfeeding at 6 mo & 46,313 & 76.1 \\
Pet owner & 14,341 & 23.6 \\
Passive smoking exposure of infants at 1 mo & 30,730 & 50.5 \\
Allergy disease & & 2.5 \\
Asthma & 1,514 & 4.4 \\
Atopic dermatitis & 2,666 & 19.5 \\
Wheezing & 11,871 & 19.0 \\
Eczema & 11,562 & SGA: sma \\
SGA: small gestational age; JPY: Japanese yen. & SGA: small gestational age; JPY: Japanese yen. \\
The $p$ value was determined using the chi-square test. & The $p$ value was determined using the chi-square test. & The $p$ val \\
\hline
\end{tabular}

Table 2. Risks of allergic diseases associated with cesarean delivery.

\begin{tabular}{llllll}
\hline & & Asthma & $\begin{array}{l}\text { Atopic } \\
\text { dermatitis } \\
\text { Ref }\end{array}$ & Wheezing & Eczema \\
$\begin{array}{l}\text { Vaginal } \\
\text { delivery }\end{array}$ & Ref & Ref & Ref \\
$\begin{array}{l}\text { Cesarean } \\
\text { delivery }\end{array}$ & $\mathrm{cOR}(95 \% \mathrm{CI})$ & $1.09(0.97-1.22)$ & $0.96(0.88-1.06)$ & $1.04(0.99-1.09)$ & $0.94(0.90-0.99)$ \\
& & & & & \\
& $\mathrm{aOR}^{* 1}(95 \%$ & 0.97 & 1.02 & 0.99 & 0.99 \\
& $\mathrm{CI})$ & $(0.85-1.10)$ & $(0.92-1.13)$ & $(0.94-1.04)$ & $(0.94-1.04)$ \\
& $\mathrm{aOR}{ }^{* 2}(95 \%$ & 0.95 & 1.02 & 0.98 & 0.99 \\
& $\mathrm{CI})$ & $(0.84-1.08)$ & $(0.92-1.13)$ & $(0.93-1.04)$ & $(0.94-1.05)$
\end{tabular}




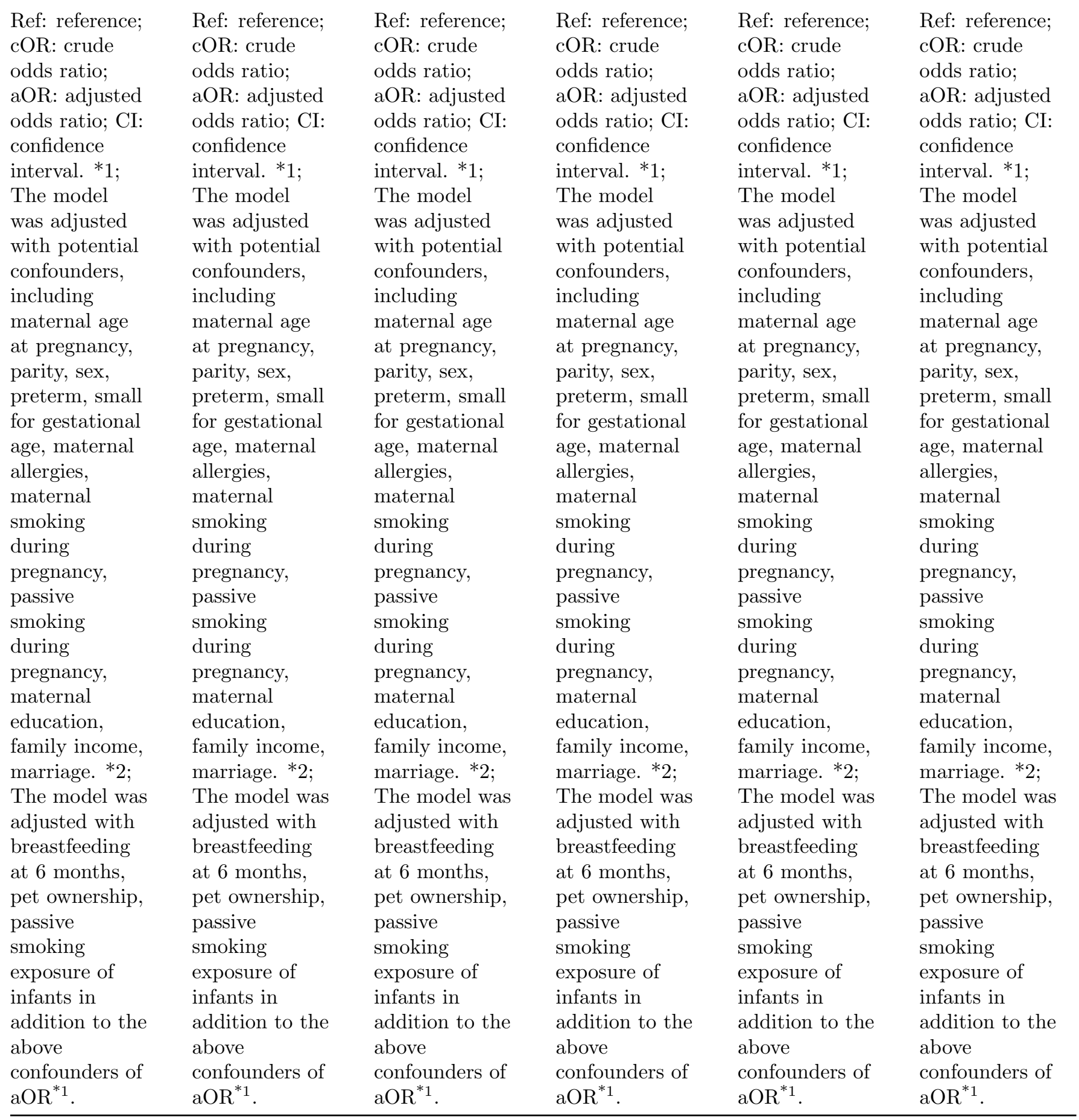

Table 3. Risks of allergic diseases associated with elective and emergent cesarean delivery. 


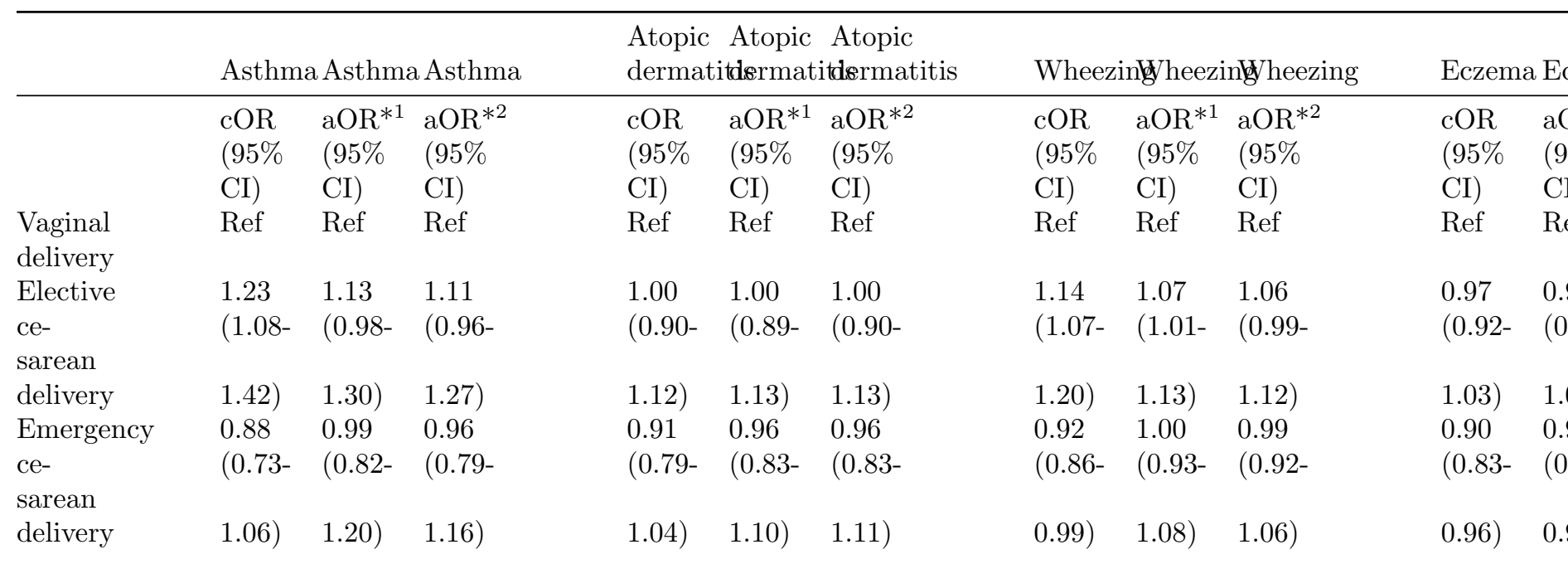




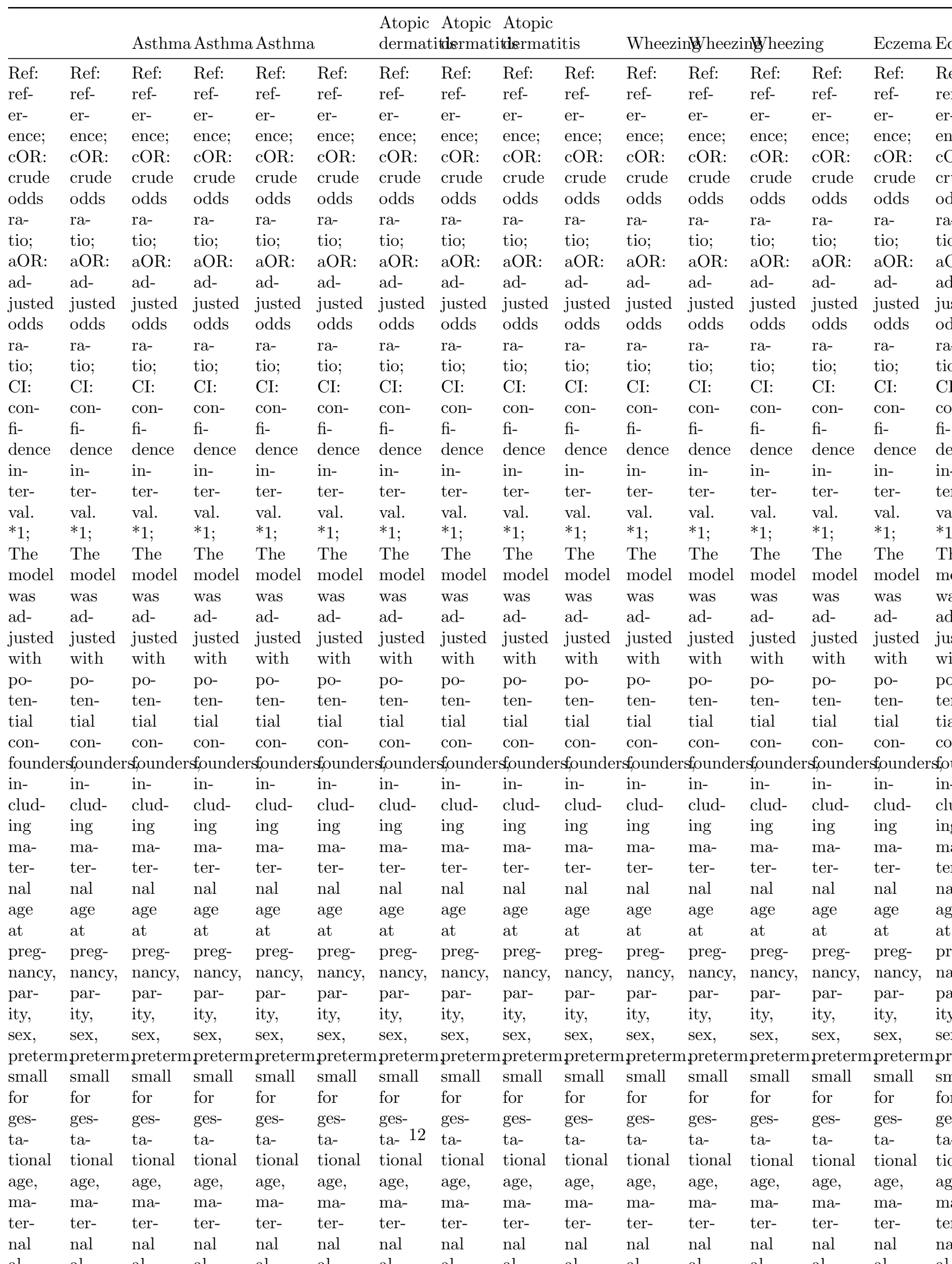


Atopic Atopic Atopic

Asthma Asthma Asthma

dermatitdermatitdermatitis

Wheezing/heezing/heezing

Eczema Ec

\section{ix. Figure legends:}

Figure 1. Flow diagram of the sample population selected for analysis.

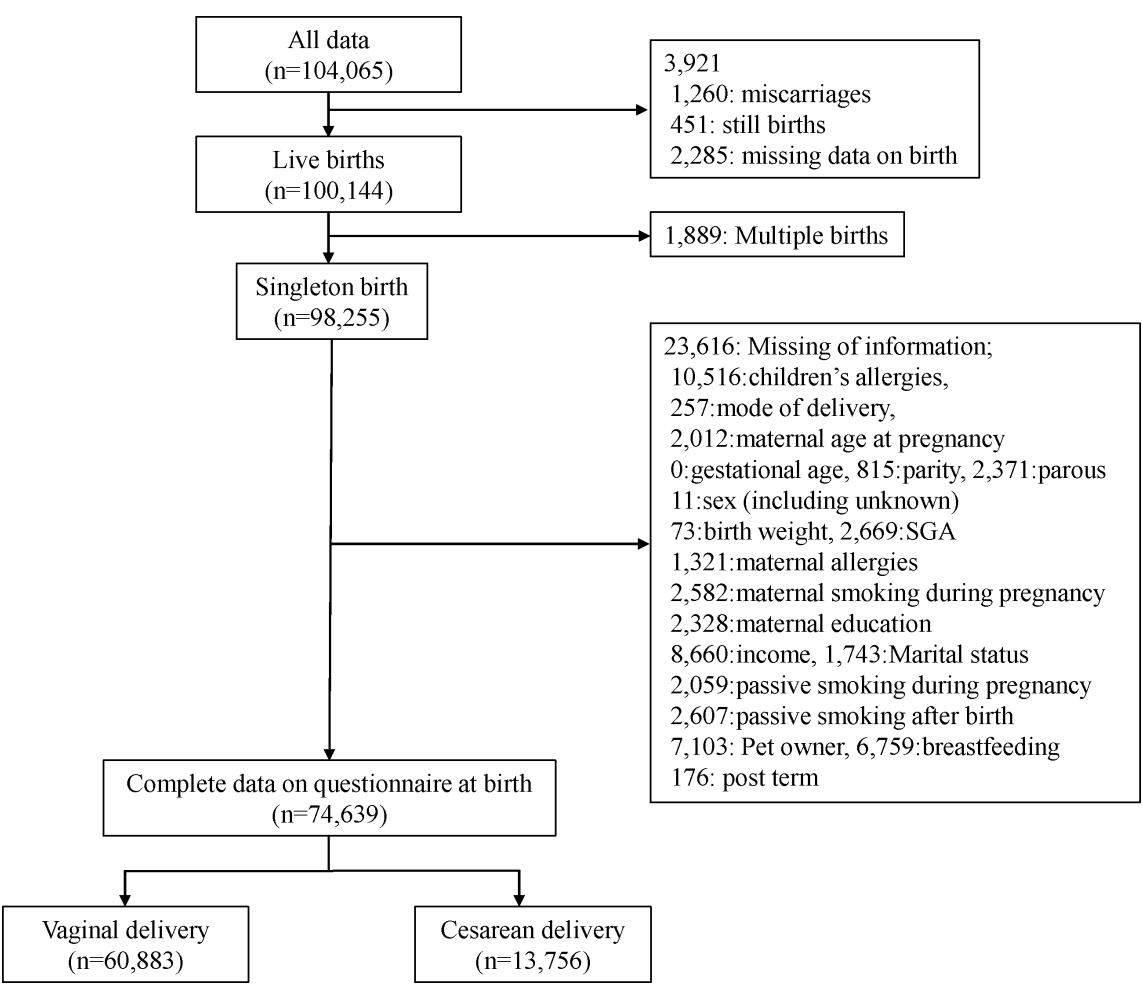

